A N N A L E S Annales de Bretagne et des Pays de l'Ouest

Anjou. Maine. Poitou-Charente. Touraine

$119-4 \mid 2012$

Varia

\title{
The Printed Book in Brittany
}

\section{Philippe Hamon}

\section{OpenEdition}

Journals

Édition électronique

URL : http://journals.openedition.org/abpo/2527

DOI : $10.4000 / a b p o .2527$

ISBN : 978-2-7535-2236-7

ISSN : 2108-6443

Éditeur

Presses universitaires de Rennes

Édition imprimée

Date de publication : 31 décembre 2012

Pagination : 149-153

ISBN : 978-2-7535-2234-3

ISSN : 0399-0826

Référence électronique

Philippe Hamon, "The Printed Book in Brittany », Annales de Bretagne et des Pays de l'Ouest [En ligne], 119-4 | 2012, mis en ligne le 31 décembre 2012, consulté le 02 mai 2019. URL : http:// journals.openedition.org/abpo/2527

Ce document a été généré automatiquement le 2 mai 2019.

(c) Presses universitaires de Rennes 


\title{
The Printed Book in Brittany
}

\author{
Philippe Hamon
}

\section{RÉFÉRENCE}

Walsby, Malcolm, The Printed Book in Brittany, 1484-1600, Leiden-Boston, Brill, 2011, 391 p., ISBN 978-90-04-20451-5.

1 Malcolm Walsby publie son deuxième livre sur l'Ouest de la France. Après The Counts of Laval (2007), il s'attaque au monde du livre en Bretagne au xvi ${ }^{e}$ siècle. Il bénéficie pour cela de l'expérience accumulée au cours de sa participation à la mise au point d'un nouvel outil bibliographique sur le livre. Avec Andrew Pettegree et Alexander S. Wilkinson, il est en effet l'un des auteurs de French vernacular books. A bibliography of books published in the French language before 1601 (Leidon-Boston, Brill, 2007), travail qui se poursuit avec la mise au point d'un outil équivalent sur les livres en latin.

2 Cette compétence lui permet de reprendre à frais nouveaux le dossier breton, en le traitant dans sa globalité, en faisant place au libraire, au commanditaire et au client à côté de l'imprimeur. Son travail 'sur une longue tradition historique et érudite, dont il fait la synthèse, mais il ne s'en contente pas. Il met au jour de nouvelles éditions et propose de nouvelles attributions comme pour les ouvrages parus entre 1526 et 1535 « Ex carracteribus Parrhisiis », qu'il attribue à un imprimeur rennais (p. 78-92). Mais surtout, par delà ses apports érudits, il expose une thèse d'ensemble. Il entend démontrer que la place limitée de l'édition en Bretagne n'est pas incompatible avec un vrai succès de l'imprimé dans la province. Et c'est en mettant en évidence des modèles économiques qu'il construit sa démonstration.

3 La première phase, au temps des incunables (ouvrages publiés avant 1500), montre la précocité de l'imprimerie bretonne, ainsi que la dispersion de ses foyers de production (5 pour un million d'habitant, contre 26 pour 14 millions dans le royaume). Les initiatives relèvent avant tout d'un patronage élitaire ponctuel, particulièrement net au tout début, à Bréhant-Loudéac, avec un cadet de la famille Rohan. Cette production de dévotion et de loisir ne construit cependant pas un modèle économique viable, faute d'un marché 
consistant. Aussi le xvi siècle est-il d'abord marqué par un net recul de la production : aucune édition à Nantes entre 1518 et 1572 ! Ce sont les libraires qui jouent alors un rôle central et $\mathrm{M}$. Walsby juge leur importance sous-estimée jusqu'ici. Très actifs, ils alimentent le marché breton en faisant imprimer des ouvrages ailleurs et en achetant à Paris ou Lyon des livres dont le coût de transport est alors très réduit, en particulier sur l'axe Lyon-Nantes. Entre 1500 et 1523, pour une centaine d'ouvrages sur lesquels figure le nom d'un membre nantais ou rennais du commerce du livre, neuf seulement sont publiés en Bretagne. Parmi les livres conservés de la bibliothèque de René de Bourgneuf, président au parlement de Bretagne mort en 1587, qui sont plus d'une centaine, pas un seul n'a été édité en Bretagne et plus de $60 \%$ l'ont été à l'étranger. M. Walsby l'affirme avec force: c'est précisément le succès du livre imprimé en Bretagne qui nuit à l'imprimerie locale car les efficaces réseaux de libraires, appuyés sur la production, à la fois de qualité et de prix raisonnable, de grands centres d'édition extérieurs, créent un environnement trop compétitif pour qu'émerge une production bretonne notable. Il est d'ailleurs significatif de voir qu'en grande majorité les auteurs «bretons » confient leurs œuvres à des éditeurs extérieurs.

4 Il existe pourtant une imprimerie bretonne, avant tout à Rennes, moins écrasée que Nantes en ce domaine. Mais, à Rennes comme ailleurs dans la province, c'est en occupant des niches précises que l'on peut survivre, voire se développer. Il faut d'abord se concentrer sur les livres qui ont un lectorat important, mais proprement breton (comme les Coutumes de Bretagne). Mais surtout, il faut pouvoir obtenir des institutions locales des travaux de faible volume, y compris de simples placards, qui garantissent une activité relativement régulière : municipalités et cours royales, parlement en tête, semblent ici jouer un rôle plus important que les commandes cléricales et universitaires. M. Walsby se penche aussi sur la survie économique d'imprimeurs protestants dans un environnement massivement catholique. Il constate des comportements différents. À Nantes en 1561, la visibilité de l'engagement calviniste de Mathurin Papolin, longtemps éditeur de textes de dévotion catholique, entraîne son départ de la ville. À Rennes en revanche, les Du Clos prennent soin de réserver leurs convictions réformées à la sphère privée et affichent publiquement une grande neutralité, ce qui autorise la poursuite de leur activité.

Une phase nouvelle s'ouvre avec la période ligueuse (1589-1598). Pour la première fois en effet, une véritable production pamphlétaire prend son envol en Bretagne. De nouveaux imprimeurs se lancent alors, à Nantes (Des Marestz) comme à Rennes (Logeroys): ils publient des textes polémiques qui émanent de prédicateurs célèbres (comme Jacques Le Bossu à Nantes) mais aussi d'institutions bretonnes divisées entre royaux et ligueurs et qui se lancent des anathèmes réciproques. Ces textes sont brefs : entre 1588 et 1598, les publications bretonnes sont en moyenne neuf fois plus courtes que pour la période antérieure (1577-1587). Avec le retour de la paix en 1598, cette nouvelle niche disparaitt et, avec elle, certains des imprimeurs tel Des Marestz. L'imprimerie locale se réorganise. Elle a finalement été rajeunie et renforcée par la phase ligueuse et elle connaît au xviie siècle un grand essor (production multipliée par six par rapport au xvie).

$6 \quad$ M. Walsby multiplie les mises au point qui servent sa démonstration. Il souligne le poids de l'édition en langue vernaculaire ( $86 \%$ ) et rappelle que pour l'essentiel les livres en langue bretonne sont imprimés hors de Bretagne. Il précise que pendant une large part de la période, l'imprimé complète, sans la remplacer totalement, la production manuscrite. Enfin, il montre la place du livre dans la province et rappelle au passage que la 
bibliothèque de Bertrand d'Argentré, avec près de 3000 titres, est l'une des plus importantes, voire la plus importante, des collections privées françaises du xvi ${ }^{\mathrm{e}}$ siècle.

7 Les copieuses annexes du livre ajoutent à son intérêt: on y trouve en effet une liste de tous les libraires et imprimeurs actifs en Bretagne pendant la période, une liste plus complète que jamais des textes imprimés en Bretagne ou pour des libraires bretons (409 items) et enfin un ensemble de dossiers sur ceux qui leur ont été attribués à tort, depuis les ordonnances des évêques de Saint-Brieuc (1496-1507) jusqu'aux éditions des Contes et discours ‘Eutrapel de Noël du Fail faussement assignées à un éditeur rennais appelé « Noël Glamet ».

On est admiratif devant la capacité de $\mathrm{M}$. Walsby à mobiliser une documentation très lacunaire et très hétérogène au service de sa démonstration: à côté des éditions disponibles, attentivement examinées et utilisées, des sources archivistiques d'une grande diversité sont mises en œuvre. Au delà des dépouillements de l'auteur, son travail n'est rendu possible que par le recours aux nombreuses chercheurs antérieures, en bonne place dans la riche bibliographie du livre. L'ouverture de ses perspectives et son angle d'attaque original lui permettent de sortir des limites, voire des impasses, d'une approche trop locale, parfois teintée de «nationalisme » breton. M. Walsby combine largeur de vue et connaissance précise du dossier pour proposer un modèle du marché du livre dont il montre en conclusion qu'il renvoie à une situation très fréquente en Europe (en comparant avec Angers et... avec l'Écosse) : par delà des nuances locales, elle serait celle de l'ensemble des régions dominées par de grands foyers éditoriaux extérieurs.

En Bretagne même, l'enquête peut encore être poursuivie. À la liste des placards publiés par M. Walsby pour la période de la Ligue, on pourra ajouter d'autres items, par exemple pour les publications «royalistes» de Michel Logeroys, mentionnées en 1589 dans les arrêts sur requête du parlement (Arch. dép. d'Ille-et-Vilaine, 1Bf, 57 et 58). Mais elles ne font que conforter le modèle proposé ici. Il faut aussi s'interroger sur le sort à faire aux plaquettes portant sur l'actualité bretonne et publiées à Lyon et Paris entre 1589 et 1598 : leur marché est-il avant tout breton, ou français?

Chez ce très bon connaisseur de la Bretagne, quelques maladresses cependant concernant le parlement : les grands jours ne sont pas créés en 1492 par François ii... mort en 1488 (p. 115); il a établi son " parlement » à Vannes dès 1485. Une phrase laisse entendre à tort que le parlement n'est pas stabilisé à Rennes dès 1561 (p. 217). Cependant, il est intéressant de noter que, sur un aspect bien plus fondamental, M. Walsby pense, comme l'auteur de ces lignes, que l'union de 1532 entraîne des réformes qui renforcent les institutions du duché et sa situation de semi-autonomie, "même si cela peut sembler contre-intuitif »(p. 114).

11 En étudiant la relation entre villes et monde du livre, $M$. Walsby perçoit dès les années 1560 une sensibilité catholique plus intolérante à Nantes et une autre plus tolérante à Rennes, à travers le rapport aux protestants. Il me semble qu'il y a ici matière à débat. La différence d'attitude peut relever d'abord du rapport de force local : on lutte plus contre les protestants à Nantes parce qu'ils y sont bien plus menaçants. Quant au conflit entre archidiacre de Nantes et parlement « rennais » (p. 197-199, avec une probable erreur de date (p.197): saisie en juillet 1561, et non 1562), il relève sans doute plus d'une concurrence institutionnelle que d'un fossé politico-religieux. D'ailleurs les parlementaires « rennais » en cause siégeaient à Nantes quelques mois plus tôt. L'auteur sous-estime enfin la force du catholicisme royal rennais qui, pendant la Ligue, limite les 
persécutions, mais n'est prêt à aucun compromis avec l'hérésie : il en donnera maintes preuves au siècle suivant.

12 Il faut conclure. En allant (p. 162) au delà des réserves exprimées par Alain Croix dans L'âge d'or de la Bretagne, M. Walsby ajoute finalement une nouvelle touche à cet âge d'or, en estimant la culture de l'imprimé dans la province bien plus importante que les constats attristés sur son imprimerie ne le laissaient penser. Certes, le caractère fragmentaire des indices peut fragiliser certaines affirmations. Parfois, l'auteur doit d'ailleurs prendre appui sur sa vision des logiques du marché plus que sur des preuves empiriques, pour affirmer la forte présence du livre en Bretagne. Mais la démonstration est utile, intelligente, souvent excitante et généralement probante. Un très bon livre. 